\title{
The CAOS Problem-Solving Environment: tools for AO numerical modeling and post-AO deconvolution
}

\author{
Marcel Carbillet ${ }^{\mathrm{a}}$ and Andrea La Camera ${ }^{\mathrm{b}}$ \\ a'Laboratoire Lagrange, Université Côte d'Azur, Observatoire de la Côte d'Azur, CNRS, Parc \\ Valrose, 06100 Nice, France \\ bDipartimento di Informatica, Bioingegneria, Robotica e Ingegneria dei Sistemi (DIBRIS), \\ Università di Genova, Via Dodecaneso 35, 16145 Genova, Italy
}

\begin{abstract}
The CAOS problem-solving environment (PSE) is an IDL-based tool developed for adaptive optics (AO) modeling and post-AO imaging. Two scientific packages enhance the CAOS PSE: the Software Package AIRY, a tool for deconvolution of post-AO images, and the eponymous Software Package CAOS, an end-to-end code for AO system simulations. In the first part of this paper we present the status and most recent developments concerning the whole CAOS PSE and its two presently developed scientific packages, while in the rest of the paper we provide two examples of application involving the Software Package AIRY: one tackling high-dynamic range images reconstruction, and the other one real data processing.
\end{abstract}

Keywords: Adaptive optics, modeling and numerical simulations, post-processing of AO data

\section{THE CAOS PSE, THE SOFTWARE PACKAGE CAOS, AND THE SOFTWARE PACKAGE AIRY}

Since its version 7.0, the CAOS Problem Solving Environment (CAOS PSE) ${ }^{1-3}$ has a unique basic distribution containing its global user interface (the so-called CAOS Application Builder ${ }^{4}$ ), its library of routines, and a package Utilities containing utility modules (to display/save/read data). The scientific packages developped beside are presently the eponymous Software Package CAOS $^{5,6}$ and the Software Package AIRY. ${ }^{7-9}$ An example of use of the global user interface of the CAOS PSE together with modules of the Software Package AIRY is shown in next section.

Within the global interface, the list of installed Software Packages is shown thanks to the pull-down menu Modules. From this list, and selecting one of installed package, its modules can be selected and placed into the so-called "worksheet" in order to compose a simulation project by combining together the modules and defining the corresponding data flow. As an example, the list of modules of package Utilities is shown in Fig. 1, while the Software Package CAOS and the Software Package AIRY are also shown as installed packages.

The IDL code implementing the simulation program is then automatically generated, at the end of the simulation project design step or at any moment, by pushing pull-down button File, and the whole structure of the simulation is saved as a project that can be restored for latter modifications and/or parameters upgrading. Last version of the CAOS PSE, 7.1, includes a number of debugging and enhancements of routines from the common library, the global user interface, and the display utility. Note that, together with their specific sets of example projects, the two scientific packages AIRY and CAOS are distributed separately.

The Software Package CAOS (Code for Adaptive Optics Systems) is dedicated to end-to-end AO simulations, and includes scientific modules for modeling optical turbulence, image formation, wavefront sensing (with both ShackHartman and pyramid wavefront sensors), wavefront reconstruction, loop control, wavefront correction, AO system calibration, laser guide stars (with upward/downward propagation and Sodium layer modeling), Fizeau interferometry, and coronagraphy. Wide-field $\mathrm{AO}$ is also developed, permitting ground-layer $\mathrm{AO}$ simulations and conjugation of deformable mirrors at different altitudes. The list of the modules of the Software Package CAOS is shown in Tab. 1.

Further author information:

M. C.: E-mail: marcel.carbillet@unice.fr

A. L. C.: E-mail: andrea.lacamera@unige.it 
Table 1. The 31 modules of the Software Package CAOS, version 7.0.

\begin{tabular}{|c|c|}
\hline Module & Purpose \\
\hline $\begin{array}{l}\text { Optical turbulence \& image formation } \\
\text { ATM - ATMosphere building } \\
\text { SRC - SouRCe definition } \\
\text { GPR - Geometrical PRopagator } \\
\text { IMG - IMaGing device }\end{array}$ & $\begin{array}{l}\text {-builds the turbulent atmosphere (FFT+subharmonics, Zernike) } \\
\text { (see also utility PSG - Phase Screen Generation) } \\
\text {-characterizes the guide star/observed object } \\
\text {-propagates light from source to telescope through atmosphere } \\
\text {-forms an image of the observed object (+ +detector noises) }\end{array}$ \\
\hline $\begin{array}{l}\text { Wavefront sensing } \\
\text { PYR - PYRamid wavefront sensor } \\
\text { SLO - SLOpe computation } \\
\text { SWS - Shack-Hartman Wavefront Sensor } \\
\text { BQC - Barycentre/Quad-cell Centroiding } \\
\text { IWS - Ideal Wavefront Sensing } \\
\text { TCE - Tip-tilt CEntroiding }\end{array}$ & $\begin{array}{l}\text {-simulates the pyramid wavefront sensor } \\
\text {-computes the slopes from the pyramid signals } \\
\text {-simulates the Shack-Hartmann (SH) wavefront sensor } \\
\text {-compute the signals from the SH spots centroiding calculus } \\
\text {-applies "ideal" wavefront sensing (see text) } \\
\text {-computes and reconstructs tip-tilt }\end{array}$ \\
\hline $\begin{array}{l}\text { Wavefront reconstruction, control \& correction } \\
\text { REC - wavefront REConstruction } \\
\text { TFL - Time-FiLtering } \\
\text { SSC - State-Space Control } \\
\text { DMI - Deformable MIrror } \\
\text { TTM - Tip-Tilt Mirror }\end{array}$ & $\begin{array}{l}\text {-reconstructs the wavefront } \\
\text {-applies time-filtering after wavefront reconstruction } \\
\text {-applies state-space control } \\
\text {-simulates the behavior of a deformable mirror (DM) } \\
\text {-simulates the behavior of a tip-tilt mirror }\end{array}$ \\
\hline $\begin{array}{l}\text { Calibration } \\
\text { CFB - Calibration FiBer characterization } \\
\text { MDS - Mirror Deformation Sequencer } \\
\text { SCD - Save Calibration Data }\end{array}$ & $\begin{array}{l}\text {-defines a fiber to be used for calibration purpose } \\
\text {-generates a sequence of DM modes or influence functions } \\
\text {-saves the calibration data (interaction matrix+set of deformates) }\end{array}$ \\
\hline $\begin{array}{l}\text { Wide-field AO } \\
\text { AVE - signals AVEraging } \\
\text { COM - COMbine measurements } \\
\text { DMC - Deformable Mirror Conjugated }\end{array}$ & $\begin{array}{l}\text {-averages measurements from various wavefront sensors } \\
\text {-combines measurements from various wavefront sensors } \\
\text {-corrects at different conjugated altitudes }\end{array}$ \\
\hline $\begin{array}{l}\text { Other modelling modules } \\
\text { LAS - LASer characterization } \\
\text { NLS - Na-Layer Spot definition } \\
\text { IBC - Interferometric Beam Combiner } \\
\text { COR - CORonagraphic module } \\
\text { AIC - Achromatic Interfero-Coronagraph } \\
\text { BSP - Beam SPlitter }\end{array}$ & $\begin{array}{l}\text {-defines laser projector characteristics } \\
\text {-characterizes the Sodium-layer behavior } \\
\text {-combines the light from two apertures } \\
\text {-simulates various coronagraphs (Lyot, Roddier\&Roddier, FQPM) } \\
\text {-simulates the Achromatic Interfero-Coronagraph } \\
\text {-splits the light beam }\end{array}$ \\
\hline $\begin{array}{l}\text { Other utility modules } \\
\text { WFA - WaveFront Adding } \\
\text { ATA - ATmosphere Adding } \\
\text { IMA - IMage Adding } \\
\text { STF - STructure Function }\end{array}$ & $\begin{array}{l}\text {-adds or combines together wavefronts } \\
\text {-adds or combines together atmospheres } \\
\text {-adds or combines together images } \\
\text {-calculates the structure function and compares to theory }\end{array}$ \\
\hline
\end{tabular}




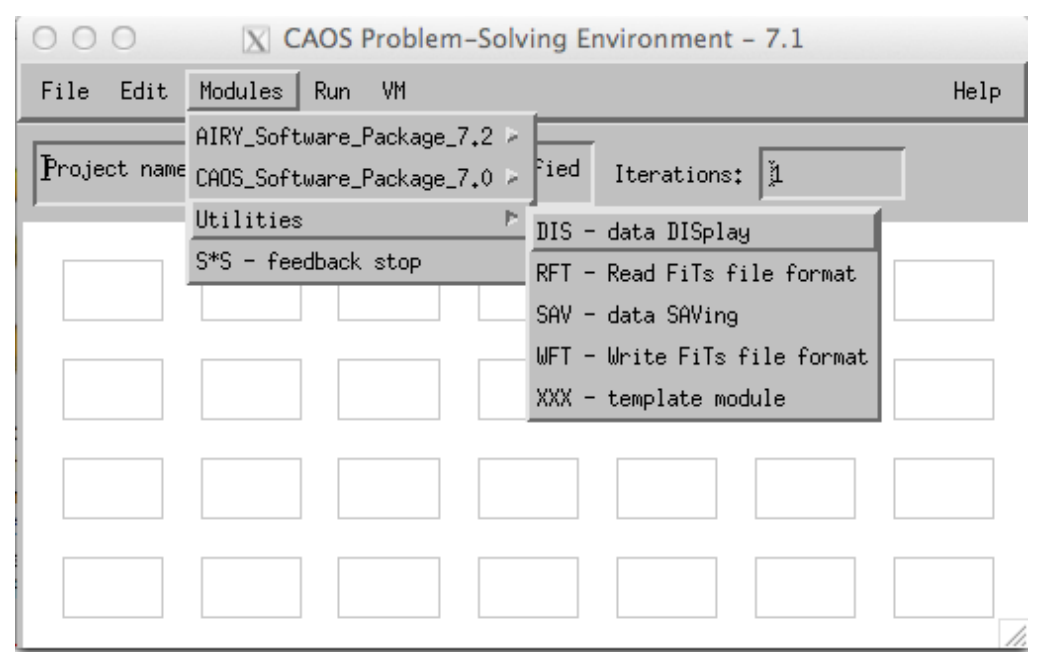

Figure 1. The global user interface of the CAOS PSE, within which the pull-down menu Modules shows the installed packages. In particular, the lists of modules of the utility package Utilities is shown.

The Software Package AIRY (Astronomical Image Restoration with or without interferometrY) now reached version 7.2, and is a complete tool for simulation and deconvolution of astronomical data (either a post-AO image from a single-dish telescope or multiple images from a Fizeau interferometer). The list of the modules of the Software Package AIRY is shown in Tab. 2.

Table 2. The 11 modules of AIRY version 7.2, divided in three subsets, depending on the goal of each module.

\begin{tabular}{l|l}
\hline Module & Purpose \\
\hline OBJ - OBJect definition & -defines the astronomical object to be convolved \\
CNV - CoNVolution & -convolves object and psf \\
ADN - ADd Noise & -adds noise perturbation to the image \\
\hline Deconvolution & -defines the background and other preprocessing data \\
PRE - PREprocessing data & -standard module for deconvolution \\
DEC - DEConvolution & -special algorithm for the deconvolution of HDR images \\
MCD - Multi-Component Deconvolution & -for blind reconstruction, if the psf is not known \\
CBD - Constrained Blind Deconvolution & -extracts the psf from an image \\
PEX - Psf extraction and EXtrapolation & -utility module, for (de)rotation of images \\
RTI - RoTate Image & \\
\hline Data Analysis & -analyses reconstructed image \\
ANB - ANalysis Binary & -analyses reconstructed image \\
FSM - Find Stars Module &
\end{tabular}

The Software Package AIRY implements several methods for reconstructing images considering Gaussian or Poisson statistics: Richardson-Lucy (RL), Ordered-Subset Expectation Maximisation (OSEM), and the Scaled Gradient Projection (SGP) method, ${ }^{10}$ with a number of regularization capabilities and a multi-component (MC) deconvolution module. ${ }^{9}$ Remarkable features of AIRY include also a super-resolution method, ${ }^{11,12}$ Strehl-constrained blind deconvolution, ${ }^{13,14}$ and high dynamic range (HDR) capabilities. ${ }^{15-17}$

An example of application of these HDR capabilities of the Software Package AIRY is detailed in next section, while an example of application on real SPHERE/VLT data of a Wolf-Rayet star in the near-infrared domain is shown in Section 3, using the SGP method with an edge-preserving regularization. Finally, foreseen developments of the whole tool are described in Section 4. 


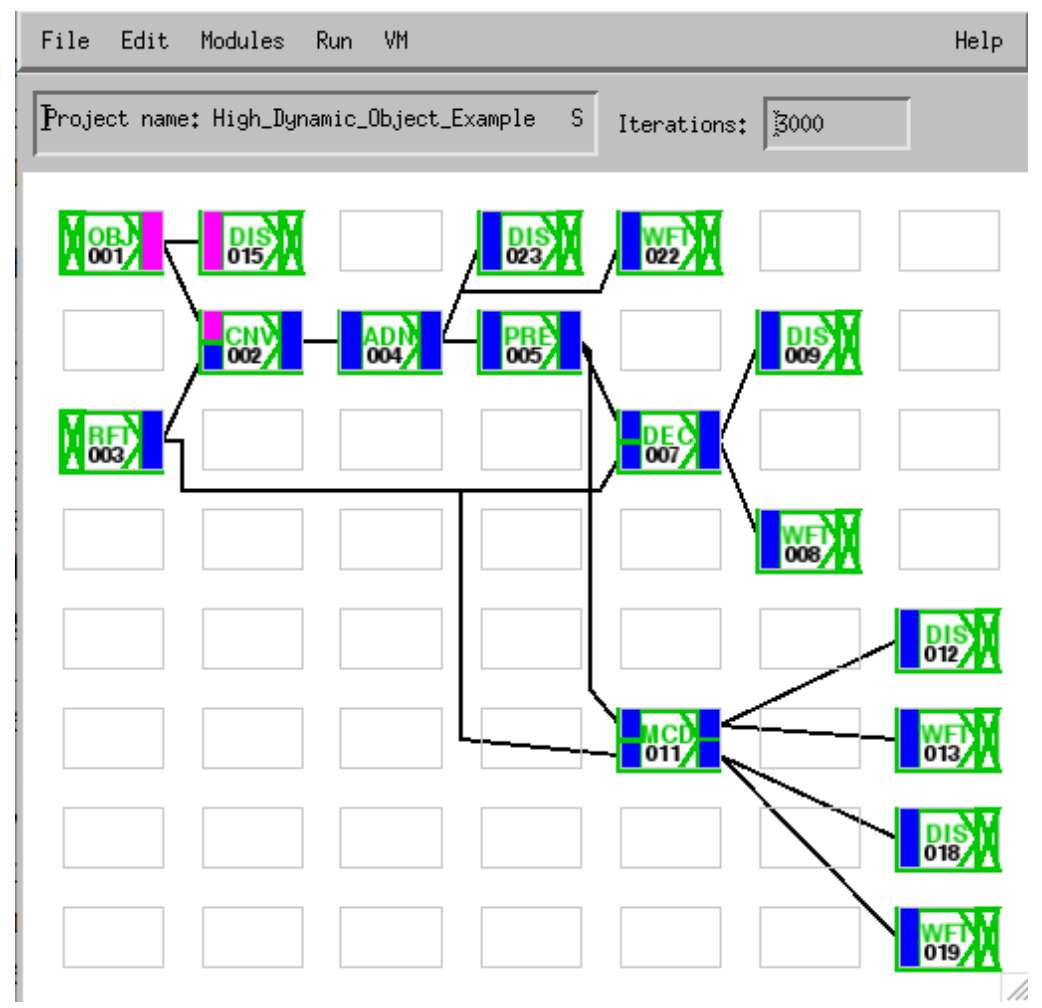

Figure 2. The AIRY project as represented within the CAOS PSE global interface.

\section{DECONVOLUTION OF HIGH-DYNAMIC RANGE IMAGES WITH AIRY}

In this example* we show two different approaches to the problem of deconvolution of high dynamic range images. The first one, already present in the previous versions of this example project, is based on a regularization version of the OSEM algorithm called HDR and introduced by Anconelli et al. in $2006 .{ }^{18}$ The new one is the multi-component method based on the Split Gradient Projection ${ }^{10}$ algorithm (in short MC-SGP), available in AIRY since version 7.0 thanks to module MCD (which stands for Multi-Component Deconvolution). The method was recently described by La Camera et al. ${ }^{9,16}$ We recall here that MC-SGP is able to reconstruct separately the unknown object assuming that it is composed of two parts: a point-like part and a diffuse part. An astronomical object made of (bright or very-bright) star(s) surrounded by a diffuse nebula is a perfect example of the input image that MCD is able to reconstruct.

The input object, the point-spread functions (PSFs) and all the parameters used in this simulation and all the parameters of the HDR regularization are described by Anconelli et al. ${ }^{18}$ In Fig. 2 the example project is shown within the CAOS PSE global interface. In Fig. 3 we show the three PSFs and the corresponding images, while in the first panel of Fig. 4 the original object (also known as ground truth) is shown.

Considering the multi-component method, the MCD module needs two parameters for the regularization function, and one supplementary image, the so-called mask, which contains information about the positions of the point-like components. The first parameter $(\delta)$ can be estimated by computing the mean value of the modulus of the gradient on the blurred and noisy image $(\bar{\delta})$. Since the regularization is applied only to the diffuse part (fainter, in general) we consider one order of magnitude less than the value of $\bar{\delta}$ : we hence assume here $\delta=5 \times 10^{4}$. The second parameter is the regularization parameter $\beta$ that can be chosen in the range of $0.1 \div 0.001$, even if larger or smaller values sometimes must be assumed. In this case we choose $\beta=0.03$. The input mask is given by assuming that the positions of the two stars are perfectly known. In real cases this information can be obtained with a first deconvolution of the image which provides a "sharp" version of the object and permits to identify the centroids of the stars with good precision. We point out that we define a broader region $(3 \times 3$ instead of the single pixel of the true object $)$ in order to leave to the algorithm the possibility of reconstructing the centroid of each star in any position around the central pixel.

${ }^{*}$ The example project here described is distributed together with the Software Package AIRY. 

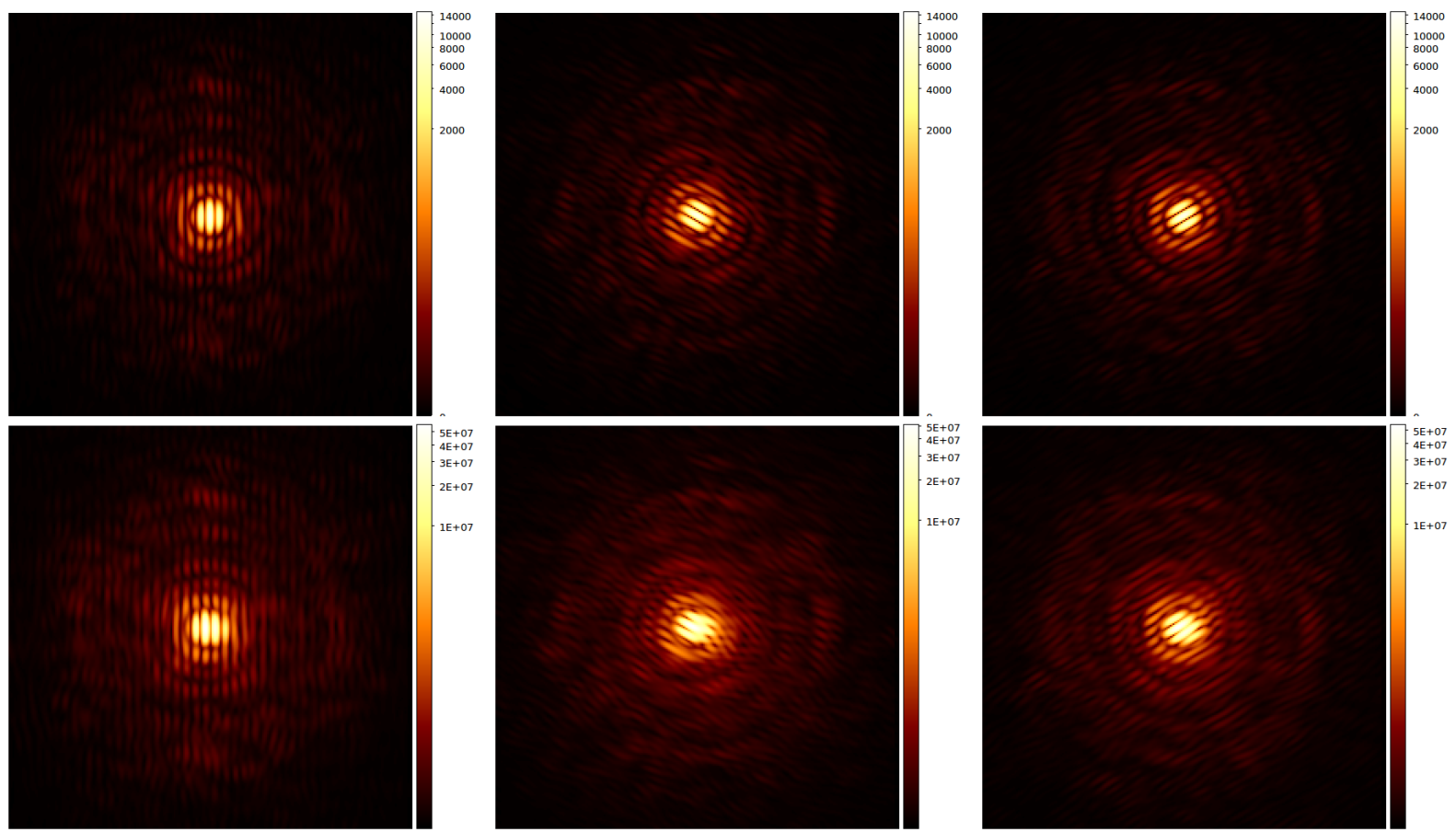

Figure 3. Top panels: the three PSFs of the Fizeau interferometer considered in the simulation. Bottom panels: the corresponding blurred and noisy images. All images are shown in logarithmic scale.
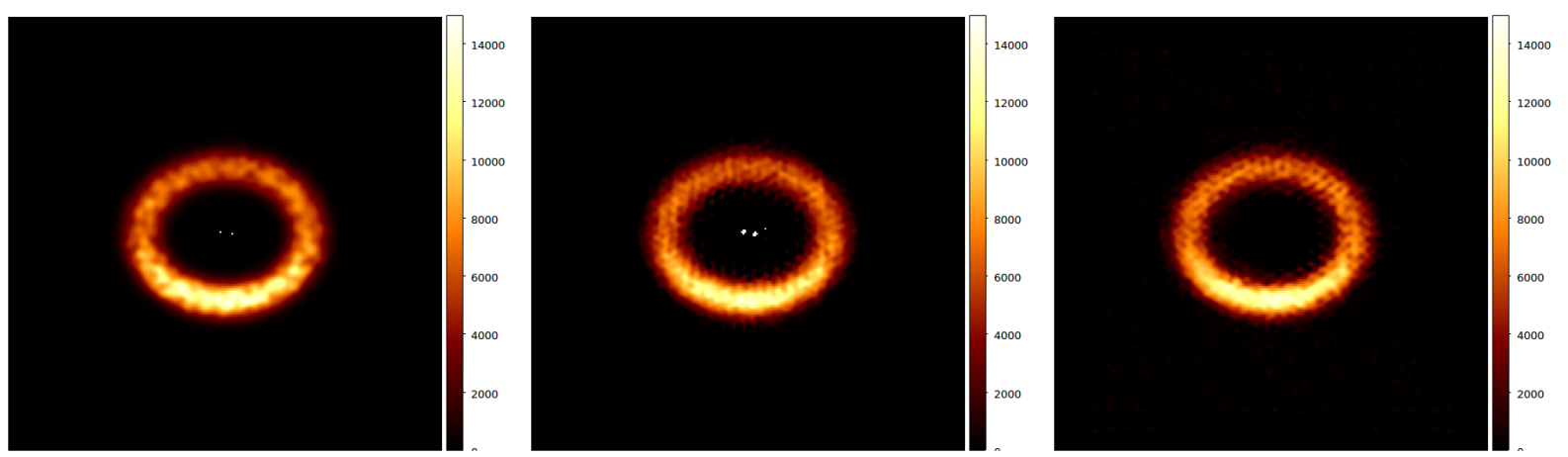

Figure 4. Left panel: the ground truth object made of two bright stars (here saturated) and a circumstellar ring. Middle panel: the reconstruction obtained by the OSEM method with the HDR regularization. Right panel: the diffuse component of the reconstruction obtained by the MC-SGP method. The point-like part is not shown. All images are shown in linear scale.

The total number of iterations is fixed to 5000 for both the methods and it is set up on the worksheet of the CAOS PSE. In Fig. 4 we show the object and the two reconstructions obtained by using the two different methods.

We evaluate the quality of the reconstructed objects thanks to two different figures of merit. For the two stars, we compute the reconstructed flux in a $3 \times 3$ pixel region, we call it $F_{n}(n=1,2)$ and we divide this flux by the true flux $F_{n}^{*}$ of each star. Concerning the diffuse part of the object we compute the so-called restoration error, defined as

$$
\rho(k)=\frac{\|f(k)-f\|}{\|f\|}
$$

where $f(k)$ is the reconstructed object at $k-t h$ iteration, $f$ is the original object and $\|\cdot\|$ is the euclidean norm. In the case of HDR reconstruction, we masked the two stars by setting to zero the $3 \times 3$ region around each star; while in the MCD reconstruction, as said above, we have the diffuse part separately reconstructed and this operation is not necessary. 

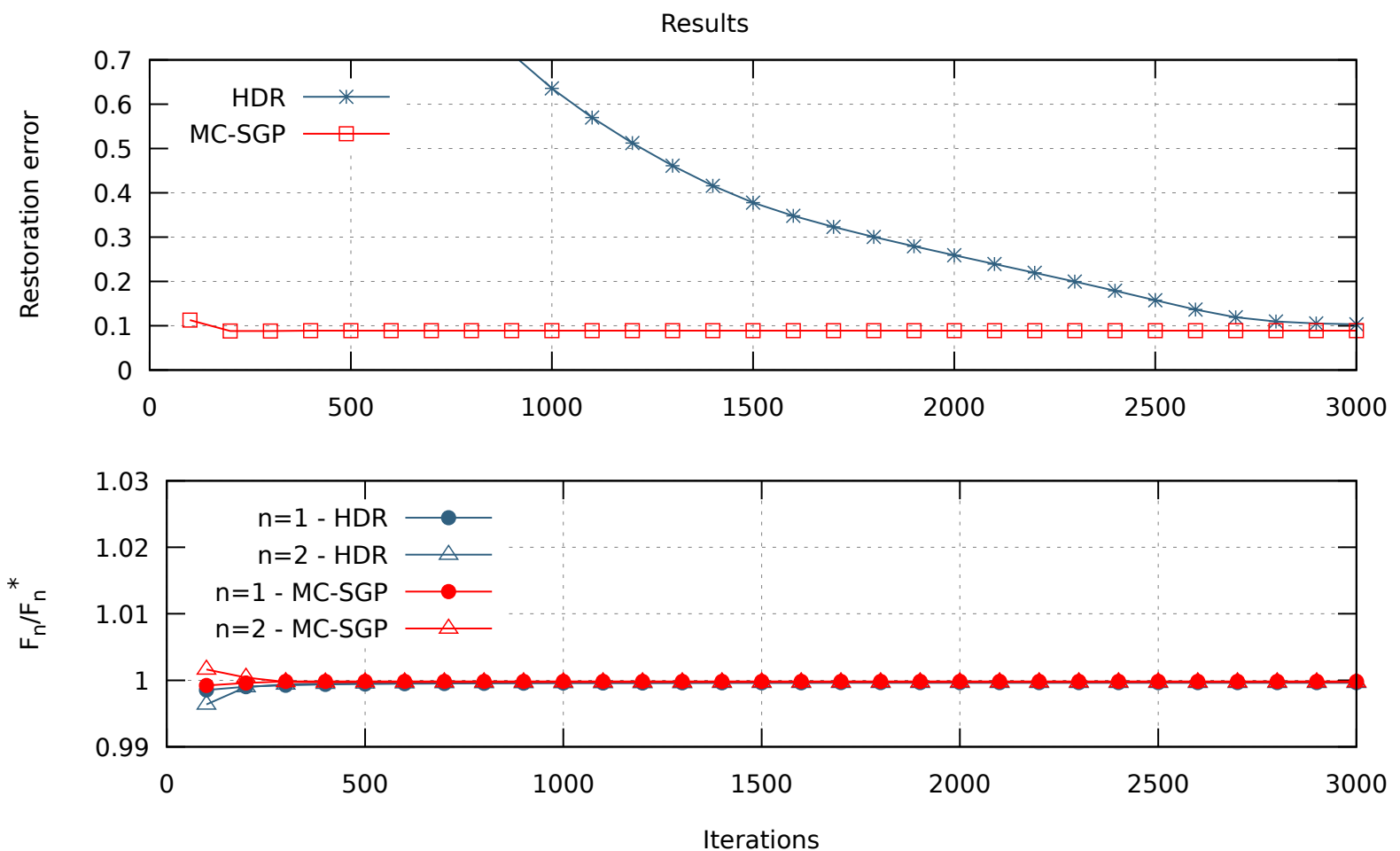

Figure 5. Top panel: the restoration error of the two reconstructions (see text). Bottom panel: the error of the fluxes as functions of the number of iterations.

Figure 5 shows the two errors as functions of the number of iterations. Concerning the first plot (top panel), we can see a different velocity in terms of convergence: as it is well-known, SGP (and therefore MC-SGP) is a faster algorithm with respect to OSEM. Moreover, the reconstruction provided by the MCD module has a smaller error (about $8.9 \%$ with respect to $10.3 \%$ of the other method). The stars are reconstructed with excellent precision (the errors on the fluxes are smaller than $1 \%$ ), and the two algorithms go rapidly here to convergence.

\section{DECONVOLUTION OF REAL DATA: WR 104 AS SEEN BY SPHERE/VLT}

A preliminary deconvolution using SGP together with an edge-preserving regularization of real data of the Wolf-Rayet star WR 104 taken in the near-infrared domain with the help of the instrument IRDIS of the planet-finder SPHERE equipping the VLT (data courtesy of A. Soulain et al.) is briefly presented here. Also thanks that SPHERE permits rather high Strehl ratios, which is the case for the K-band data presented in Fig. 6 (left panel for the image, middle panel for the associated PSF), the reconstruction performed very clearly reveals the spirale shape of the observed object (see right panel).

With the pipeline-reduced data used here, we attempt a preliminary reconstruction involving the SGP deconvolution method together with an edge-reserving regularization. As shown in Fig. 6, the result is very convincing, clearly revealing the spirale shape of the circumstellar environment of WR 104. More refined reconstructions for various wavelength bands and analysis details will be given in a forthcoming paper currently under preparation. ${ }^{19}$

\section{CURRENT APPLICATIONS AND FURTHER DEVELOPMENTS}

Concerning the Software Package CAOS, it will next include (for its version 7.1) an updated simple embedment of the semi-analytic AO code PAOLA. ${ }^{20}$ New modules for wide-field AO modeling will hopefully be finalized, in order to permit to consider multiple sources and multiple sensors within single modules, and hence an easier modeling of GLAO systems, as well as layer-oriented and star-oriented multi-conjugate AO systems. For its part, the Software 

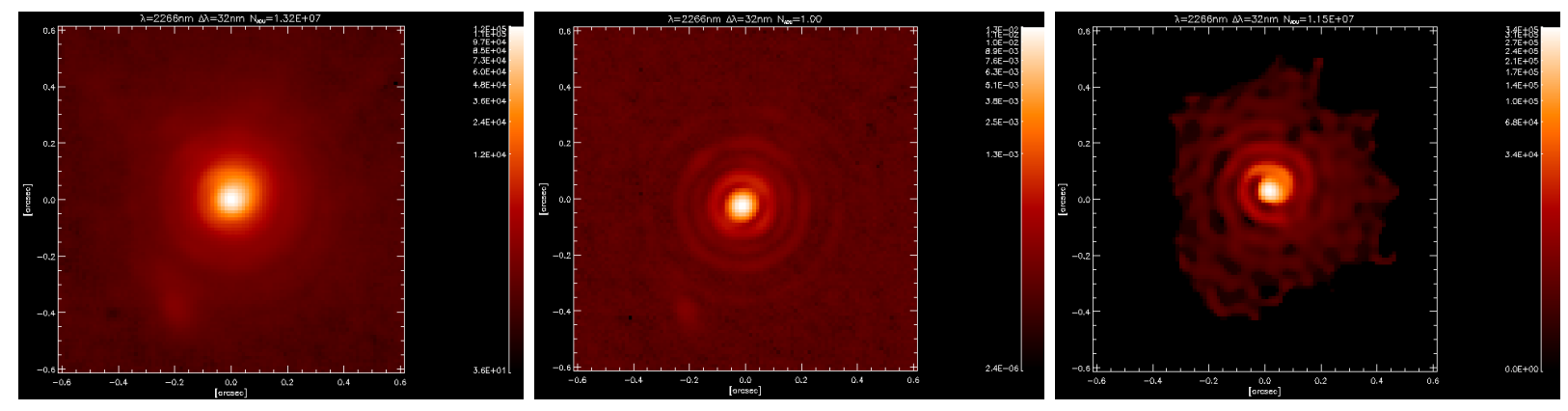

Figure 6. Left panel: observed object ( $\mathrm{K}$ band). Middle panel: associated PSF. Right panel: reconstruction with SGP and an edge-preserving regularization (data: courtesy A. Soulain et al., more details to be given in a forthcoming paper ${ }^{19}$ ).

Package AIRY is being used in a number of astrophysical applications involving post-AO images obtained from various instruments (NACO/VLT, SPHERE/VLT, LMIRcam/LBTI, NIRC2/Keck-II).

Freely download the CAOS PSE, together with the Software Package CAOS and/or the Software Package AIRY from http://lagrange.oca.eu/caos

\section{REFERENCES}

[1] Carbillet, M., Vérinaud, C., Guarracino, M., Fini, L., Lardière, O., Roux, B. L., Puglisi, A. T., Femenía, B., Anconelli, A. R. B., Bertero, M., and Boccacci, P., "CAOS - a numerical simulation tool for astronomical adaptive optics (and beyond)," in [Advancements in Adaptive Optics], Bonaccini, D., Ellerbroek, B., and Ragazzoni, R., eds., Proc. SPIE 5490 (2), 550 (2004).

[2] Carbillet, M., Desiderà, G., Augier, E., La Camera, A., Riccardi, A., Boccaletti, A., Jolissaint, L., and Kadir, D. A., "The CAOS problem-solving environment: recent developments," in [Adaptive Optics Systems II], Ellerbroek, B., Hart, M., Hubin, N., and Wizinowich, P. L., eds., Proc. SPIE 7736, 773644 (2010).

[3] "The CAOS PSE website." http://lagrange.oca.eu/caos. (last visited: October 12, 2017).

[4] Fini, L., Carbillet, M., and Riccardi, A., "The CAOS Application Builder," Astronomical Data Analysis Software and Systems X 238, 253 (2001).

[5] Carbillet, M., Vérinaud, C., Femenía, B., Riccardi, A., and Fini, L., "Modelling astronomical adaptive optics - I. The software package CAOS," Monthly Notices of the Royal Astronomical Society 356(4), 1263-1275 (2005).

[6] Carbillet, M., La Camera, A., and et al., "The Software Package CAOS 7.0: enhanced modelling of astronomical adaptive optics systems," in [Adaptive Optics Systems V], Marchetti, E., Close, L. M., and Véran, J.-P., eds., Proc. SPIE 9909, 9909-319 (2016).

[7] Correia, S., Carbillet, M., Boccacci, P., Bertero, M., and Fini, L., "Restoration of interferometric images: I. the software package AIRY," Astron. Astrophys. 387, 733-743 (2002).

[8] "The AIRY project website." http://www. airyproject.eu. (last visited: October 12, 2017).

[9] La Camera, A., Carbillet, M., Prato, M., Boccacci, P., and Bertero, M., "The software package AIRY 7.0: new efficient deconvolution methods for post-adaptive optics data," 99097T, International Society for Optics and Photonics (jul 2016).

[10] Bonettini, S., Zanella, R., and Zanni, L., "A scaled gradient projection method for constrained image deblurring," Inverse Probl. 25(1), 015002 (2009).

[11] Anconelli, B., Bertero, M., Boccacci, P., and Carbillet, M., "Restoration of interferometric images. IV. An algorithm for super-resolution of stellar systems," Astron. Astroph. 431, 747-755 (2005).

[12] Carbillet, M., La Camera, A., Chesneau, O., Millour, F., Girard, J., and Prato, M., "Deconvolution-based super resolution for post-AO data," in [Proceedings of the Third AO4ELT Conference. Firenze], 104 (2013). 
[13] Desiderà, G. and Carbillet, M., "Strehl-constrained iterative blind deconvolution for post-adaptive-optics data," Astron. Astrophys. 507(3), 1759-1762 (2009).

[14] Carbillet, M., La Camera, A., Deguignet, J., Prato, M., Bertero, M., Aristidi, E., and Boccacci, P., "Strehlconstrained reconstruction of post-adaptive optics data and the Software Package AIRY, v. 6.1," in [Adaptive Optics Systems IV], Marchetti, E., Close, L. M., and Véran, J.-P., eds., Proc. SPIE 9148, 91484 U (2014).

[15] La Camera, A., Antonucci, S., Bertero, M., Boccacci, P., Lorenzetti, D., and Nisini, B., "Image reconstruction for observations with a high dynamic range: LINC-NIRVANA simulations of a stellar jet," in [Optical and Infrared Interferometry III], Delplancke, F., Rajagopal, F. J. K., and Malbet, F., eds., Proc. SPIE 8455, 84553D (2012).

[16] La Camera, A., Antoniucci, S., Bertero, M., Boccacci, P., Lorenzetti, D., Nisini, B., and Arcidiacono, C., "Reconstruction of high dynamic range images: Simulations of lbt observations of a stellar jet, a pathfinder study for future ao-assisted giant telescopes," Publications of the Astronomical Society of the Pacific 126(936), pp. 180-193 (2014).

[17] Antoniucci, S., La Camera, A., Nisini, B., Giannini, T., Lorenzetti, D., Paris, D., and Sani, E., "The HH34 outflow as seen in [Fe II] $1.64 \mu \mathrm{m}$ by LBT-LUCl," Astron. Astrophys. 566, A129 (jun 2014).

[18] Anconelli, B., Bertero, M., Boccacci, P., Desiderà, G., Carbillet, M., and Lantéri, H., "Deconvolution of multiple images with high dynamic range and an application to LBT LINC-NIRVANA," Astron. Astrophys. 460(1), 349355 (2006).

[19] Soulain, A. et al. in preparation (2017).

[20] Jolissaint, L., "Synthetic modeling of astronomical closed loop adaptive optics," J. of the European Opt. Soc. Rapid Pub. 5, 10055-10070 (2010). 